\title{
Refinements of Kantorovich type, Schwarz and Berezin number inequalities
}

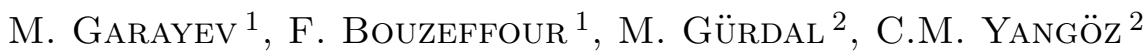 \\ ${ }^{1}$ Department of Mathematics, College of Science, King Saud University \\ P.O. Box 2455, Riyadh 11451, Saudi Arabia \\ ${ }^{2}$ Suleyman Demirel University, Department of Mathematics, 32260, Isparta, Turkey \\ mgarayev@ksu.edu.sa, fbouzaffour@ksu.edu.sa, gurdalmehmet@sdu.edu.tr, \\ mustafa_yangoz@hotmail.com
}

Abstract: In this article, we use Kantorovich and Kantorovich type inequalities in order to prove some new Berezin number inequalities. Also, by using a refinement of the classical Schwarz inequality, we prove Berezin number inequalities for powers of $f(A)$, where $A$ is self-adjoint operator on the Hardy space $H^{2}(\mathbb{D})$ and $f$ is a positive continuous function. Some related questions are also discussed.

Key words: Reproducing kernel Hilbert space, Berezin symbol, Berezin number, Kantorovich type inequality, $C^{*}$-module.

AMS Subject Class. (2010): Primary 47A63; Secondary 26D15, 47B10.

\section{Introduction, NOtATION AND PRELIMINARIES}

In 1948, L.V. Kantorovich [24] proved the following inequality

$$
\langle A x, x\rangle\left\langle A^{-1} x, x\right\rangle \leq \frac{\left(\lambda_{1}+\lambda_{n}\right)^{2}}{4 \lambda_{1} \lambda_{n}}
$$

where $x=\left(x_{1}, \ldots, x_{n}\right)$ is a unit vector in $\mathbb{C}^{n}$ and $A$ is an $n \times n$ positive-definite matrix with eigenvalues $\lambda_{1} \geq \cdots \geq \lambda_{n}>0$.

The Kantorovich inequality is still valid for an operator $A$ acting on an infinite dimensional Hilbert space $H$ with $M I \geq A \geq m I>0$ as follows:

$$
\langle A x, x\rangle\left\langle A^{-1} x, x\right\rangle \leq \frac{(M+m)^{2}}{4 m M} \quad(x \in H,\|x\|=1) .
$$

Replacing $x$ by $\frac{A^{1 / 2} x}{\left\|A^{1 / 2} x\right\|}$ in the above inequality, we get the following equivalent form of Kantorovich inequality:

$$
\left\langle A^{2} x, x\right\rangle \leq \frac{(M+m)^{2}}{4 m M}\langle A x, x\rangle^{2} \quad(x \in H,\|x\|=1) .
$$


The Kantorovich inequality is a useful tool in numerical analysis and statistics for establishing the rate of convergence of the method of steepest descent. During the past decades several formulations, extensions or refinements of the Kantorovich inequality in various settings have been introduced by many mathematicians; see, for instance, Moslehian [34] and references therein. The first generalization of the Kantorovich inequality is due to Greub and Rheinboldt [20]. They proved that if $A$ is a bounded linear operator on $H$ (i.e., $A \in \mathcal{B}(H))$ such that $M I \geq A \geq m I>0$, then

$$
\langle x, x\rangle^{2} \leq\langle A x, x\rangle\left\langle A^{-1} x, x\right\rangle \leq\langle x, x\rangle^{2} \frac{(M+m)^{2}}{4 m M}
$$

for any $x \in H$. They also showed that inequality 1.3 is equivalent to

$$
\langle A x, A x\rangle\langle B x, B x\rangle \leq\langle A x, B x\rangle^{2} \frac{\left(M M^{\prime}+m m^{\prime}\right)^{2}}{4 m m^{\prime} M M^{\prime}},
$$

when $B$ is a selfadjoint operator permutable with $A, x \in H$ and $M^{\prime} I \geq B \geq$ $m^{\prime} I>0$. In what follows, Strang 38 generalized inequality $(1.3)$ by proving that if $A \in \mathcal{B}(H)$ is invertible, $\|A\|=M$ and $\left\|A^{-1}\right\|=\frac{1}{m}$, then

$$
\left|\left(\langle A x, x\rangle\left\langle y, A^{-1} y\right\rangle\right)\right| \leq \frac{(M+m)^{2}}{4 m M}\langle x, x\rangle\langle y, y\rangle
$$

for all $x, y \in H$ and that the bound is the best possible. For further generalization of the Kantorovich inequality and recent developments of the operator Kantorovich inequality, we refer the readers to the excellent book of Furuta [14] and Moslehian's paper [34. Note that Dragomir [10] gave several Kantorovich type inequalities involving norms and numerical radii for operators on a Hilbert space. Also, Garayev [19] and Başaran et al. [6] applied the Kantorovich inequality to get Berezin number inequalities for the operators acting on the reproducing kernel Hilbert space.

In the present article, we use some Kantorovich type inequalities to prove some new inequalities for the Berezin number of operators. Also, we apply a refinement of classical Schwarz inequality due to Dragomir [11] to prove Berezin number inequalities between some powers of $f(A)$, where $f: J \rightarrow$ $[0,+\infty)$ is a positive continuous function, where $J \subset[0,+\infty)$ and $A$ is a selfadjoint operator on the Hardy space $H^{2}(\mathbb{D})$ with spectrum in $J$. Before giving our results, we need to some definitions and notations.

Throughout this paper, $\mathcal{B}(H)$ stand for the Banach algebra of all bounded linear operators acting on a Hilbert space $(H,\langle.,\rangle$.$) . An operator A \in \mathcal{B}(H)$ is 
said to be positive if $\langle A x, x\rangle \geq 0$ for all $x \in H$. Moreover, if $A$ is invertible, we call it strictly positive and write $A>0$. A reproducing kernel Hilbert space is a Hilbert space $\mathcal{H}=\mathcal{H}(\Omega)$ of complex-valued functions on a (nonempty) set $\Omega$, which has the property that point evaluation $f \rightarrow f(\lambda)$ is continuous on $\mathcal{H}$ for all $\lambda \in \Omega$. Then the Riesz representation theorem guarantees that for every $\lambda \in \Omega$ there is a unique element $k_{\lambda}=k(., \lambda) \in \mathcal{H}$ such that $f(\lambda)=\left\langle f, k_{\lambda}\right\rangle$ for all $f \in \mathcal{H}$. The function $k_{\lambda}$ is called the reproducing kernel of $\mathcal{H}$ and the function $\widehat{k}_{\lambda}:=\frac{k_{\lambda}}{\left\|k_{\lambda}\right\|_{\mathcal{H}}}$ is the normalized reproducing kernel in $\mathcal{H}$ (see Aronzajn [3]). For any operator $A \in \mathcal{B}(\mathcal{H})$, its Berezin symbol $\widetilde{A}$ is defined by (see Berezin [7, 8])

$$
\widetilde{A}(\lambda):=\left\langle A \widehat{k}_{\lambda}, \widehat{k}_{\lambda}\right\rangle, \quad \lambda \in \Omega .
$$

The Berezin number of $A$ is defined as

$$
\operatorname{ber}(A):=\sup \{|\mu|: \mu \in \operatorname{Ber}(A)\}
$$

where

$$
\operatorname{Ber}(A)=\operatorname{Range}(\widetilde{A})=\{\widetilde{A}(\lambda): \lambda \in \Omega\}
$$

is the so-called Berezin set of operator $A$ (see Karaev [25]). The numerical range and numerical radius of the operator $A$ is defined, respectively, by

$$
W(A):=\{\langle A x, x\rangle: x \in H \text { and }\|x\|=1\}
$$

and

$$
w(A):=\sup \{|\langle A x, x\rangle|: x \in H \text { and }\|x\|=1\} .
$$

Clearly, $|\widetilde{A}(\lambda)| \leq\|A\|$, and more precisely, $\operatorname{ber}(A) \leq w(A) \leq\|A\|$. Note that the celebrated Berger-Halmos-Pearcy inequality for the powers of the operator $A \in \mathcal{B}(H)$ is the following (see Halmos [22] and Pearcy [37], and references therein):

$$
w\left(A^{n}\right) \leq w(A)^{n}, \quad n=1,2, \ldots .
$$

Since $\operatorname{ber}(A) \leq w(A)$, it is natural to ask about the same inequalities for the Berezin number ber $(A)$, i.e., is it true that

$$
\operatorname{ber}\left(A^{n}\right) \leq \operatorname{ber}(A)^{n} \quad \text { for any integer } n \geq 1 \text { ? }
$$

However, Coburn proved in his paper 9 that for some concrete operator $X$ on the Bergman space $L_{a}^{2}(\mathbb{D})$ of analytic functions on $\mathbb{D}$, $\operatorname{ber}\left(X^{2}\right)>\operatorname{ber}(X)^{2}$, which shows that Berger-Halmos-Pearcy theorem fails for $\operatorname{ber}(A)$. But, still it 
is interesting to investigate inverse estimates $\operatorname{ber}(A)^{n} \leq C \operatorname{ber}(A)^{n}, n \geq 1$, and also other type Berezin number inequalities between the powers of operators.

In this article, motivated mainly by the paper [34, we will prove some new inequalities for the Berezin number of powers of operators by using Kantorovich and Kantorovich type inequalities and also a refinement of Schwarz inequality due to Dragomir [10]. For the related results, the reader can see in [4, 5, 13, 15, 16, 17, 18, 21, 25, 26, 30, 32, 33, 36, 40, 41, 42, 44]

\section{Some Corollaries of Kantorovich inequalities}

In this section, we give some immediate corollaries of several Kantorovich type inequalities which entail new inequalities for Berezin numbers of some operator classes. First, we start with Strang inequality (1.4).

Proposition 1. If $A \in \mathcal{B}(\mathcal{H}(\Omega))$ is invertible, $\|A\|=M$ and $\left\|A^{-1}\right\|=$ $m^{-1}$, then

$$
\operatorname{ber}(A) \operatorname{ber}\left(A^{-1}\right) \leq \frac{(M+m)^{2}}{4 m M}
$$

Proof. In fact, by putting in inequality (1.4) $x=\widehat{k}_{\lambda}, y=\widehat{k}_{\mu}(\lambda, \mu \in \Omega)$, we have

$$
\left|\left\langle A \widehat{k}_{\lambda}, \widehat{k}_{\lambda}\right\rangle\right|\left|\left\langle\widehat{k}_{\mu}, A^{-1} \widehat{k}_{\mu}\right\rangle\right| \leq \frac{(M+m)^{2}}{4 m M}
$$

or equivalently,

$$
|\widetilde{A}(\lambda)|\left|\widetilde{A^{-1 *}}(\mu)\right|=|\widetilde{A}(\lambda)|\left|\widetilde{A^{-1}}(\mu)\right| \leq \frac{(M+m)^{2}}{4 m M}
$$

for all $\lambda, \mu \in \Omega$. This implies immediately the required inequality (2.1).

The following is immediate from the inequality (1.2).

Proposition 2. If $A \in \mathcal{B}(\mathcal{H}(\Omega))$ is an operator such that $M I \geq A \geq$ $m I>0$, then

$$
\operatorname{ber}\left(A^{2}\right) \leq \frac{(M+m)^{2}}{4 m M} \operatorname{ber}(A)^{2} .
$$

The next result follows from Furuta's inequality [13] which is an extension of the Kantorovich inequality. 
Proposition 3. If $A, B \in \mathcal{B}(\mathcal{H})$ are positive operators, $A \geq B>0$ and $M I \geq B \geq m I>0$, then

$$
\begin{aligned}
\left(\frac{M}{m}\right)^{p-1} & \operatorname{ber}\left(A^{p}\right) \\
& \geq \frac{(p-1)^{p-1}}{p^{p}}\left(\frac{\left(M^{p}-m^{p}\right)^{p}}{(M-m)\left(m M^{p}-M m^{p}\right)^{p-1}}\right) \operatorname{ber}\left(A^{p}\right) \\
& \geq \operatorname{ber}\left(B^{p}\right)
\end{aligned}
$$

holds for each $p \geq 1$; the constant

$$
k_{+}(m, M, p)=\frac{(p-1)^{p-1}}{p^{p}}\left(\frac{\left(M^{p}-m^{p}\right)^{p}}{(M-m)\left(m M^{p}-M m^{p}\right)^{p-1}}\right)
$$

is called the Ky Fan-Furuta constant in the literature (see, for instance, [13] and its references).

Proof. In fact, by the well-known Furuta's inequality

$$
\left(\frac{M}{m}\right)^{p-1} A^{p} \geq k_{+}(m, M, p) A^{p} \geq B^{p} \quad \text { for each } p \geq 1
$$

or equivalently,

$$
\left(\frac{M}{m}\right)^{p-1}\left\langle A^{p} x, x\right\rangle \geq k_{+}(m, M, p)\left\langle A^{p} x, x\right\rangle \geq\left\langle B^{p} x, x\right\rangle
$$

for all $x \in H$. In particular, for $x=\widehat{k}_{\lambda}$ and for all $\lambda \in \Omega$, we obtain that

$$
\left(\frac{M}{m}\right)^{p-1} \widetilde{A^{p}}(\lambda) \geq k_{+}(m, M, p) \widetilde{A^{p}}(\lambda) \geq \widetilde{B^{p}}(\lambda) .
$$

Then, by taking supremum in these inequalities, we obtain the desired inequality (2.2).

The following is a corollary of Furuta's result in ([14, Theorem 1.1]).

Proposition 4. Let $A \in \mathcal{B}(\mathcal{H})$ be a self-adjoint operator on a reproducing kernel Hilbert space $H=\mathcal{H}(\Omega)$ satisfying $M I \geq A \geq m I>0$. Then

$$
\operatorname{ber}\left(A^{p}\right) \leq \frac{\left(m M^{p}-M m^{p}\right)}{(q-1)(M-m)}\left(\frac{(q-1)\left(M^{p}-m^{p}\right)}{q\left(m M^{p}-M m^{p}\right)}\right)^{q} \operatorname{ber}(A)^{q}
$$

under anyone of the following conditions (i) and (ii) respectively: 
(i) $m^{p-1} q \leq \frac{M^{p}-m^{p}}{M-m} \leq M^{p-1} q$ holds for real numbers $p>1$ and $q<0$;

(ii) $m^{p-1} q \leq \frac{M^{p}-m^{p}}{M-m} \leq M^{p-1} q$ holds for real numbers $p<0$ and $q<0$.

First, we cite the following lemma [14] to give a proof of this proposition.

Lemma 1. Let $h(t)$ be defined by

$$
h(t)=\frac{1}{t^{q}}\left(k+\frac{K-k}{M-m}(t-m)\right)
$$

on $[m, M](M>m>0)$, where $q$ is any real number such that $q \neq 0,1$ and $K$ and $k$ are any real numbers. Then $h(t)$ has the following upper bound on $[m, M]$ :

$$
\frac{(m K-M k)}{(q-1)(M-m)}\left(\frac{(q-1)(K-k)}{q(m K-M k)}\right)^{q}
$$

where $m, M, k, K$ and $q$ in (2.4) satisfy anyone of the following conditions (i) and (ii) respectively:

(i) $K>k, \frac{K}{M}>\frac{k}{m}$ and $\frac{k}{m} q \leq \frac{K-k}{M-m} \leq \frac{K}{M} q$ holds for a real number $q>1$;

(ii) $K<k, \frac{K}{M}<\frac{k}{m}$ and $\frac{k}{m} q \leq \frac{K-k}{M-m} \leq \frac{K}{M} q$ holds for a real number $q<0$.

Proof. It is elementary that $h^{\prime}\left(t_{0}\right)=0$ when

$$
t_{0}=\frac{q}{q-1} \frac{m K-M k}{K-k}
$$

and it turns out that to satisfies the required condition $t_{0} \in[m, M]$ and also to gives the upper bound (2.4) of $h(t)$ on the segment $[m, M]$ under any one of the conditions (i) and (ii) respectively.

Proof of Proposition 4. Since $f(t)$ is a real-valued continuous convex function on $[m, M]$, we have

$$
f(t) \leq f(m)+\frac{f(M)-f(m)}{M-m}(t-m) \quad \text { for any } t \in[m, M] .
$$

Then by passing to the operational calculus of positive operator $A$ in 2.5 ) since $M \geq\left\langle A \widehat{k}_{\lambda}, \widehat{k}_{\lambda}\right\rangle \geq m$, we obtain for every $\lambda \in \Omega$ that

$$
\left\langle f(A) \widehat{k}_{\lambda}, \widehat{k}_{\lambda}\right\rangle \leq f(m)+\frac{f(M)-f(m)}{M-m}\left(\left\langle A \widehat{k}_{\lambda}, \widehat{k}_{\lambda}\right\rangle-m\right),
$$


or equivalently

$$
\widetilde{f(A)}(\lambda) \leq f(m)+\frac{f(M)-f(m)}{M-m}(\widetilde{A}(\lambda)-m) .
$$

Multiplying $(\widetilde{A}(\lambda))^{-q}$ on both sides of $(2.6)$, we get

$$
(\widetilde{A}(\lambda))^{-q} \widetilde{f(A)}(\lambda) \leq h(t)
$$

where

$$
h(t)=(\widetilde{A}(\lambda))^{-q}\left(f(m)+\frac{f(M)-f(m)}{M-m}\right)(\widetilde{A}(\lambda)-m) .
$$

Then we obtain that

$$
\widetilde{f(A)}(\lambda) \leq\left(\max _{m \leq t \leq M} h(t)\right)(\widetilde{A}(\lambda))^{q} .
$$

Substituting $K=f(M)$ and $k=f(m)$ in [34, Theorem 1.1], then (i) and (ii) in Theorem 1.1 just correspond to (i) and (ii) in Lemma 1. We have from (2.8) that

$$
\operatorname{ber}(f(A)) \leq\left(\max _{m \leq t \leq M} h(t)\right)(\operatorname{ber}(A))^{q} .
$$

Now put $f(t)=t^{p}$ for $p \notin[0,1]$ in $(2.9)$. Since $f(t)$ is a real-valued continuous convex function on $[m, M], M^{p}>m^{p}$ and $M^{p-1}>m^{p-1}$ hold for any $p>1$, that is, $f(M)>f(m)$ and $\frac{f(M)}{M}>\frac{f(m)}{m}$ for any $p>1$ and also $M^{p}<m^{p}$ and $M^{p-1}<m^{p-1}$ hold for any $p<0$, that is $f(M)<f(m)$ and $\frac{f(M)}{M}<\frac{f(m)}{m}$ for any $p<0$ respectively. Whence the proof is complete by $(2.9)$.

Our next result, associated with Hölder-McCarthy and Kantorovich inequalities, is the following.

Proposition 5. Let $A$ be positive operator on a Hilbert space $H=\mathcal{H}(\Omega)$ satisfying $M I \geq A \geq m I>0$. Then the following inequality holds for every $\lambda \in \Omega$ :

(i) In case $p>1$ : $\operatorname{ber}(A)^{p} \leq \operatorname{ber}\left(A^{p}\right) \leq K_{+}(m, M) \operatorname{ber}(A)^{p}$, where

$$
K_{+}(m, M)=\frac{(p-1)^{p-1}}{p^{p}} \frac{\left(M^{p}-m^{p}\right)^{p}}{(M-m)\left(m M^{p}-M m^{p}\right)^{p-1}} .
$$


(ii) In case $p<0$ : $\operatorname{ber}(A)^{p} \leq \operatorname{ber}\left(A^{p}\right) \leq K_{-}(m, M) \operatorname{ber}(A)^{p}$, where

$$
K_{-}(m, M)=\frac{\left(m M^{p}-M m^{p}\right)}{(p-1)(M-m)}\left(\frac{(p-1)\left(M^{p}-m^{p}\right)}{p\left(m M^{p}-M m^{p}\right)}\right) .
$$

Proof. As $f(t)=t^{p}$ is a convex function for $p \notin[0,1]$, (i) and (ii) Proposition 4 hold in case $p \notin[0,1]$ and $q \neq p$, so that the inequalities of the right-hand sides of (i) and (ii) hold by Proposition 4 and ones of the left-hand sides of (i) and (ii) follow by Hölder-McCarthy inequality [14.

Corollary 1. Let $A \in \mathcal{B}(\mathcal{H})$ be a positive operator on a reproducing kernel Hilbert space $\mathcal{H}=\mathcal{H}(\Omega)$ such that $M I \geq A \geq m I>0$. Then:

(i) $\sup _{\lambda \in \Omega}\left[(\widetilde{A}(\lambda))^{p} \widetilde{A^{-1}}(\lambda)\right] \leq \frac{p^{p}}{(p+1)^{p+1}} \frac{(m+M)^{p+1}}{m M}$;

(ii) $\operatorname{ber}\left(A^{2}\right) \leq \frac{p^{p}}{(p+1)^{p+1}} \frac{(m+M)^{p+1}}{(m M)^{p}}(\operatorname{ber}(A))^{p+1}$ for any $p \in\left[\frac{m}{M}, \frac{M}{m}\right]$.

Proof. (i) In (ii) of Proposition 4, we have only to put $p=-1$ and replacing $q$ by $-p$ for $p>0$.

(ii) In (i) in Proposition 4 , we have only to put $p=2$ and replacing $q$ by $p+1$ for $p>0$. This proves the corollary.

\section{A Berezin number inequality Via the VARIANCE-COVARIANCE INEQUALITY}

Following [34], we give some necessary concepts and notations. The notion of semi-inner product $C^{*}$-module is a generalization of that of semi-inner product space in which the semi-inner product takes its values in a $C^{*}$-algebra instead of the field of complex numbers. We can define a semi-norm on a semiinner product $(\mathcal{X},\langle.,\rangle$.$) over a C^{*}$-algebra $\mathcal{A}$ by $\|x\|=\|\langle x, x\rangle\|^{\frac{1}{2}}$, where the latter norm denotes that of $\mathcal{A}$. $A$ pre-Hilbert $\mathcal{A}$-module (or an inner-product module) is a semi-inner product module in which $\|$.$\| defined as above is a$ norm. If this norm is complete then $\mathcal{X}$ is called a Hilbert $C^{*}$-module. Each $C^{*}$ algebra $\mathcal{A}$ can be regarded as a Hilbert $\mathcal{A}$-module via $\langle a, b\rangle=a^{*} b(a, b \in \mathcal{A})$. When $\mathcal{X}$ is a Hilbert $C^{*}$-module, we denote by $\mathbb{B}(\mathcal{X})$ the $C^{*}$-algebra of all adjointable operators on $\mathcal{X}$. For every $x \in \mathcal{X}$ the absolute value of $x$ is defined by $|x|=\langle x, x\rangle^{1 / 2} \in \mathcal{A}$. Some standard references for $C^{*}$-modules are [2, 29, 31]. 
In this section, we present some Kantorovich inequalities for Berezin symbols of operators involving unital positive linear mappings and the operator geometric mean in the framework of semi-inner product $C^{*}$-modules and get some new Berezin number inequalities. Let $x, y, z, x_{1}, \ldots, x_{n}$ be arbitrary elements of a semi-inner product $\mathcal{A}$-module $(\mathcal{X},\langle.,\rangle$.$) . In [34, the authors$ studied the covariance

$$
\operatorname{cov}_{z}(x, y):=\|z\|^{2}\langle x, y\rangle-\langle x, z\rangle\langle z, y\rangle
$$

and the variance

$$
\operatorname{var}_{z}(x):=\operatorname{cov}_{z}(x, x),
$$

and proved that $\left[\operatorname{cov}_{z}\left(x_{i}, x_{j}\right)\right] \in M_{n}(\mathcal{A})$ is positive, or equivalently

$$
\|z\|^{2}\left[\left\langle x_{i}, x_{j}\right\rangle\right] \geq\left[\left\langle x_{i}, z\right\rangle\left\langle z, x_{j}\right\rangle\right] ;
$$

this is called Generalized Covariance-Variance Inequality. In particular, by the Cauchy-Schwarz inequality for the semi-inner product $\operatorname{cov}_{z}(.,$.$) , the$ Covariance-Variance inequality holds

$$
\operatorname{cov}_{z}(x, y) \operatorname{cov}_{z}(x, y)^{*} \leq\left\|\operatorname{var}_{z}(y)\right\| \operatorname{var}_{z}(x) .
$$

Let $\mathcal{A}$ be a $C^{*}$-algebra and $\mathcal{B}$ be a $C^{*}$-subalgebra of $\mathcal{A}$. $A$ linear mapping $\Phi: \mathcal{A} \rightarrow \mathcal{B}$ is called a (right) multiplier if $\Phi(a b)=\Phi(a) b(a \in \mathcal{A}, b \in \mathcal{B})$. If $\Phi$ is a positive multiplier, any semi-inner product $\mathcal{A}$-module $\mathcal{X}$ becomes a semi-inner product $\mathcal{B}$-module with respect to

$$
[x, y]_{\Phi}:=\Phi(\langle x, y\rangle) \quad(x, y \in \mathcal{X})
$$

By (3.1), it holds

$$
\|\Phi(\langle z, z\rangle)\|\left[\Phi\left(\left\langle x_{i}, x_{j}\right\rangle\right)\right] \geq\left[\Phi\left(\left\langle x_{i}, z\right\rangle\right) \Phi\left(\left\langle z, x_{j}\right\rangle\right)\right]
$$

for all $z, x_{1}, \ldots, x_{n} \in \mathcal{X}$. In the sequel, we will assume that the $\mathcal{A}$-module $\mathcal{X}$ is a reproducing kernel Hilbert space over some suitable set $\Omega$ with the normalized reproducing kernel $\widehat{k}_{\lambda}(z)=\frac{k_{\lambda}(z)}{\left\|k_{\lambda}\right\|_{\chi}}, \lambda, z \in \Omega$. So, if we fix a normalized reproducing kernel $\widehat{k}_{\lambda} \in \mathcal{X}$ and take operators $A$ and $B$ in $\mathbb{B}(\mathcal{X})$, then we can define the $\lambda$-covariance of $A, B$ and $\lambda$-variance of $A$ by

$$
\lambda-\operatorname{cov}(A, B)=\Phi\left(\left\langle A \widehat{k}_{\lambda}, B \widehat{k}_{\lambda}\right\rangle\right)-\Phi\left(\left\langle A \widehat{k}_{\lambda}, \widehat{k}_{\lambda}\right\rangle\right) \Phi\left(\left\langle\widehat{k}_{\lambda}, B \widehat{k}_{\lambda}\right\rangle\right)
$$


and

$$
\lambda-\operatorname{var}(A)=\lambda-\operatorname{cov}(A, A),
$$

respectively, similar to Enomoto [12]. Our idea is mainly related to the work of Umegaki [43] and Moslehian [34] where, in particular, the proofs of several known inequalities for Hilbert space operators are unified.

Lemma 2. Let $\mathcal{A}$ be a unital $C^{*}$-algebra and $\mathcal{B}$ be a unital $C^{*}$-subalgebra of $\mathcal{A}$ such that both algebras have the same unit e. Let $\mathcal{X}=\mathcal{X}(\Omega)$ be a $\mathcal{A}$-module consisting from the reproducing kernel Hilbert space of complexvalued functions on $\Omega$ with the normalized reproducing kernel $\widehat{k}_{\lambda}$. Let $A_{1}, A_{2} \in$ $\mathbb{B}(\mathcal{X})$ be two operators satisfying $m_{i} I \leq A_{i} \leq I M_{i}$ for some scalars $m_{i}, M_{i}$ $(i=1,2)$. Then

$$
\sup _{\lambda \in \Omega}\left\|\lambda-\operatorname{cov}\left(A_{1}, A_{2}\right)\right\| \leq \frac{1}{4}\left(M_{1}-m_{1}\right)\left(M_{2}-m_{2}\right) .
$$

Proof. It can be easily seen that

$$
(M-C)(C-m) \leq\left(\frac{M-m}{2}\right)^{2}
$$

for any self-adjoint operator $C$ of a unital $C^{*}$-algebra with spectrum in $[m, M]$, due to $\left(C-\frac{M+m}{2}\right)^{2} \geq 0$. Hence

$$
\begin{aligned}
\lambda-\operatorname{var}\left(A_{1}\right)= & \lambda-\operatorname{cov}\left(A_{1}, A_{1}\right) \\
= & \Phi\left(\left\langle A_{1}^{2} \widehat{k}_{\lambda}, \widehat{k}_{\lambda}\right\rangle\right)-\Phi\left(\left\langle A_{1} \widehat{k}_{\lambda}, \widehat{k}_{\lambda}\right\rangle\right)^{2} \\
= & \left(\left(M_{1} e-\Phi\left(\left\langle A_{1} \widehat{k}_{\lambda}, \widehat{k}_{\lambda}\right\rangle\right)\right) \Phi\left(\left\langle A_{1} \widehat{k}_{\lambda}, \widehat{k}_{\lambda}\right\rangle\right)-m_{1} e\right) \\
& -\Phi\left(\left\langle\left(M_{1}-A_{1}\right)\left(A_{1}-m_{1}\right) \widehat{k}_{\lambda}, \widehat{k}_{\lambda}\right\rangle\right) \\
\leq & \left(M_{1}-\Phi\left(\left\langle A_{1} \widehat{k}_{\lambda}, \widehat{k}_{\lambda}\right\rangle\right)\right)\left(\Phi\left(\left\langle A_{1} \widehat{k}_{\lambda}, \widehat{k}_{\lambda}\right\rangle\right)-m_{1}\right) \\
\leq & \frac{1}{4}\left(M_{1}-m_{1}\right)^{2}
\end{aligned}
$$

(by $\left(M_{1}-A_{1}\right)\left(A_{1}-m_{1}\right) \geq 0$, the selfadjointness of $\Phi\left(\left\langle A_{1} \widehat{k}_{\lambda}, \widehat{k}_{\lambda}\right\rangle\right)$ and the positivity of the Berezin symbol $\widetilde{A}_{1}$ of a positive operator $A_{1}$ ). So, we have that

$$
\lambda-\operatorname{var}\left(A_{1}\right) \leq \frac{1}{4}\left(M_{1}-m_{1}\right)^{2},
$$


and similarly

$$
\lambda-\operatorname{var}\left(A_{2}\right) \leq \frac{1}{4}\left(M_{2}-m_{2}\right)^{2}
$$

for all $\lambda \in \Omega$. From these inequalities, by using the covariance-variance inequality, we have for all $\lambda \in \Omega$ that

$$
\begin{aligned}
\left\|\lambda-\operatorname{cov}\left(A_{1}, A_{2}\right)\right\|^{2} & \leq\left\|\lambda-\operatorname{var}\left(A_{1}\right)\right\|\left\|\lambda-\operatorname{var}\left(A_{2}\right)\right\| \\
& \leq \frac{1}{16}\left(M_{1}-m_{1}\right)^{2}\left(M_{2}-m_{2}\right)^{2},
\end{aligned}
$$

which gives the required inequality.

Our next result is the " $\lambda$-parametrization" variant of Moslehian's result ([34, Theorem 2.2]) about generalization of Kantorovich inequality (see also [41]).

Theorem 1. Let $\mathcal{A}$ be a unital $C^{*}$-algebra and $\mathcal{B}$ be a unital $C^{*}$-subalgebra of $\mathcal{A}$ such that both have the same unit e. Let $\mathcal{X}=\mathcal{X}(\Omega)$ be a $\mathcal{A}$ module consisting of the reproducing kernel Hilbert space of complex-valued functions on $\Omega$ with reproducing kernel $k_{\lambda}$. Let $A \in \mathbb{B}(\mathcal{X})$ be an operator satisfying $m I \leq A \leq M I$ for some scalars $0<m<M$. Then

$$
\sup _{\lambda \in \Omega}\left|\Phi(\widetilde{A}(\lambda)) \Phi\left(\widetilde{A^{-1}}(\lambda)\right)\right| \leq \frac{(M+m)^{2}}{4 M m} .
$$

Proof. Put $A_{1}=A, A_{2}=A^{-1}, m_{1}=m, M_{1}=M, m_{2}=M^{-1}, M_{2}=m^{-1}$ in Lemma 2 to get

$$
\left|\Phi(e)-\Phi\left(\left\langle A \widehat{k}_{\lambda}, \widehat{k}_{\lambda}\right\rangle\right) \Phi\left(\left\langle A^{-1} \widehat{k}_{\lambda}, \widehat{k}_{\lambda}\right\rangle\right)\right| \leq \frac{(M-m)^{2}}{4 m M}
$$

that is

$$
\left|\Phi(e)-\Phi(\widetilde{A}(\lambda)) \Phi\left(\widetilde{A^{-1}}(\lambda)\right)\right| \leq \frac{(M-m)^{2}}{4 m M}
$$

whence

$$
\left|\Phi(\widetilde{A}(\lambda)) \Phi\left(\widetilde{A^{-1}}(\lambda)\right)\right| \leq 1+\frac{(M-m)^{2}}{4 m M}=\frac{(M+m)^{2}}{4 M m}
$$

for all $\lambda \in \Omega$, which yields 3.2 . 
Corollary 2. Let $\Phi: \mathbb{B}(\mathcal{X}) \rightarrow \mathbb{B}(\mathcal{X})$ be a unital positive linear map. If $A \in \mathbb{B}(\mathcal{X})$ is an operator satisfying $0<m I \leq A \leq M I$ for some scalars $m, M$ then

$$
\sup _{\lambda \in \Omega}\left[\widetilde{A}(\lambda) \widetilde{A^{-1}}(\lambda)\right] \leq \frac{(M+m)^{2}}{4 M m} .
$$

Proof. The proof is immediate from Theorem 1. Indeed, take $\mathcal{A}=\mathcal{B}=\mathbb{C}$. The only positive linear mapping $\Phi: \mathbb{C} \rightarrow \mathbb{C}$ is the identity mapping. For any $\lambda \in \Omega$ and $\widehat{k}_{\lambda}=\frac{k_{\lambda}}{\left\|k_{\lambda}\right\|}$, we obtain (3.3) from 3.2 .

\section{A Kantorovich inequality Via the operator Geometric MEAN AND A BEREZIN NUMBER INEQUALITY}

In this section, we use $\lambda$-parametrization for proving a generalization of the Kantorovich inequality in the context of Hilbert $C^{*}$-modules which can be viewed as extension of Theorem 1 and an inequality due to Nakamoto and Nakamura [35].

Recall that for positive invertible elements $a, b \in \mathcal{A}$, we can use the following characterization of operator mean due to Ando [1] as follows

$$
a \sharp b=\max \left\{x \in \mathcal{A}: x=x^{*},\left[\begin{array}{ll}
a & x \\
x & b
\end{array}\right] \geq 0\right\},
$$

where $a \sharp b=a^{\frac{1}{2}}\left(a^{-\frac{1}{2}} b a^{-\frac{1}{2}}\right)^{\frac{1}{2}} a^{\frac{1}{2}}$. This is easily obtained from $a=(a \sharp b) b^{-1}(a \sharp b)$ and the fact that $a \geq x b^{-1} x^{*}$ if and only if $\left[\begin{array}{cc}a & x \\ x^{*} & b\end{array}\right] \geq 0$, where $x \in \mathcal{A}$.

Theorem 2. Let $\mathcal{A}, \mathcal{B}$ be unital $C^{*}$-algebras, $\mathcal{X}=\mathcal{X}(\Omega)$ be a reproducing kernel Hilbert space with reproducing kernel $k_{\lambda}$ which is an $\mathcal{A}$-module and $A \in \mathbb{B}(\mathcal{X})$ such that $M I \geq A \geq m I>0$ for some scalars $m, M$. Then, for every $f \in \mathcal{X}$ for which $\langle f, f\rangle$ is invertible and every positive linear mapping $\Phi: \mathcal{A} \rightarrow \mathcal{B}$ it holds:

$$
\begin{aligned}
\sup _{\lambda \in \Omega} \Phi\left(\left\langle\widehat{k}_{\lambda}, \widehat{k}_{\lambda}\right\rangle\right) & \leq \sup _{\lambda \in \Omega} \Phi(\widetilde{A}(\lambda)) \# \Phi\left(\widetilde{A^{-1}}(\lambda)\right) \\
& \leq \frac{M+m}{2 \sqrt{m M}} \sup _{\lambda \in \Omega} \Phi\left(\left\langle\widehat{k}_{\lambda}, \widehat{k}_{\lambda}\right\rangle\right) .
\end{aligned}
$$

Proof. The proof is based in the similar proof of [34, Theorem 3.1]. Indeed, first note that for any $\lambda \in \Omega,\left\langle\widehat{k}_{\lambda}, \widehat{k}_{\lambda}\right\rangle$ is invertible. Therefore, for $a:=$ 
$m M\left\langle A^{-1} \widehat{k}_{\lambda}, \widehat{k}_{\lambda}\right\rangle$ and $b:=\left\langle A \widehat{k}_{\lambda}, \widehat{k}_{\lambda}\right\rangle, a \geq m\left\langle\widehat{k}_{\lambda}, \widehat{k}_{\lambda}\right\rangle$ and $b \geq m\left\langle\widehat{k}_{\lambda}, \widehat{k}_{\lambda}\right\rangle$, so $a$ and $b$ are positive and invertible. Since $\Phi$ is positive and unital, $\Phi(a)$ and $\Phi(b)$ are also positive and invertible. Observe now that $M-A$ and $\frac{1}{m}-A^{-1}$ are positive commuting elements of the $C^{*}$-algebra $\mathbb{B}(\mathcal{X})$, which implies that $(M-A)\left(\frac{1}{m}-A^{-1}\right) \geq 0$. Hence

$$
m M A^{-1}+A \leq(m+M) .
$$

Then, for every $\lambda \in \Omega$,

$$
m M\left\langle A^{-1} \widehat{k}_{\lambda}, \widehat{k}_{\lambda}\right\rangle+\left\langle A \widehat{k}_{\lambda}, \widehat{k}_{\lambda}\right\rangle \leq(m+M)\left\langle\widehat{k}_{\lambda}, \widehat{k}_{\lambda}\right\rangle
$$

from which we have

$$
m M \Phi\left(\left\langle A^{-1} \widehat{k}_{\lambda}, \widehat{k}_{\lambda}\right\rangle\right)+\Phi\left(\left\langle A \widehat{k}_{\lambda}, \widehat{k}_{\lambda}\right\rangle\right) \leq(m+M) \Phi\left(\left\langle\widehat{k}_{\lambda}, \widehat{k}_{\lambda}\right\rangle\right) .
$$

\section{Refinement of Schwartz inequality and BEREZIN NUMBER INEQUALITY}

In his paper [11], Dragomir obtained some new improvements of classical Schwarz inequality in complex Hilbert space $H$ as follows.

Lemma 3. Let $x, y, e \in H$ with $\|e\|=1$. Then we have the following refinement of Schwarz inequality

$$
\|x\|^{2}\|y\|^{2}-|\langle x, y\rangle|^{2} \geq\left(\operatorname{det}\left[\begin{array}{cc}
|\langle x, e\rangle| & \left(\|x\|^{2}-|\langle x, e\rangle|^{2}\right)^{\frac{1}{2}} \\
|\langle y, e\rangle| & \left(\|y\|^{2}-|\langle y, e\rangle|^{2}\right)^{\frac{1}{2}}
\end{array}\right]\right)^{2} .
$$

Recall that for $A \in \mathcal{B}(\mathcal{H})$, its Crawford number $c(A)$ is defined by

$$
c(A):=\inf \{|\langle A x, x\rangle|: x \in H \text { and }\|x\|=1\} .
$$

In [39], the authors introduced the numbers $\widetilde{b}(A)$ and $\widetilde{c}(A)$ defined by

$$
\widetilde{c}(A):=\inf _{\lambda \in \Omega}|\widetilde{A}(\lambda)| \quad \text { and } \quad \widetilde{b}(A):=\inf _{\lambda \in \Omega} \frac{|\widetilde{A}(\lambda)|}{\left\|A \widehat{k}_{\lambda}\right\|} .
$$


It is easy to see that $\widetilde{b}(A) \leq 1$ and $c(A) \leq \widetilde{c}(A) \leq \operatorname{ber}(A)$. These, and other related numerical characteristics, are useful in studying bounded linear operators (see [39] and its references). Note that the Hardy space $H^{2}=$ $H^{2}(\mathbb{D})$ over the disc $\mathbb{D}=\{z \in \mathbb{C}:|z|<1\}$ is the RKHS with the normalized reproducing kernel $\widehat{k}_{\lambda}(z):=\frac{\left(1-|\lambda|^{2}\right)^{1 / 2}}{1-\bar{\lambda} z}, \lambda, z \in \mathbb{D}$. For definitions and more facts about this space, we recommend the book of Hoffman [23. In this section, by applying Lemma 3, we prove Berezin number inequality. Namely, we prove the following theorem.

Theorem 3. If $f, g: J \rightarrow(0,+\infty)$ are continuous functions on some segment $J \subset[0,+\infty)$, then

(i) $\widetilde{f^{2}(A)}(\lambda)+\widetilde{g^{2}(B)}(\mu)+\widetilde{2 f^{4}(A)}(\lambda) \widetilde{g^{4}(B)}(\mu)+$

$$
\begin{aligned}
& +\widetilde{f^{3}(A)}(\lambda) \widetilde{g^{3}(B)}(\mu) \widetilde{\left(I-f^{2}(A)\right)^{\frac{1}{2}}}(\lambda) \widetilde{\left(I-g^{2}(B)\right)^{\frac{1}{2}}}(\mu) \\
\geq & \widetilde{g^{2}(B)}(\mu) \widetilde{f^{4}(A)}(\lambda)+\widetilde{g^{4}(B)}(\mu) \widetilde{f^{2}(A)}(\lambda)
\end{aligned}
$$

for any self-adjoint operators $A, B \in \mathcal{B}\left(H^{2}\right)$ with spectra in $J$ such that $I-f^{2}(A)$ and $I-g^{2}(B)$ are positive and for all $\lambda, \mu \in \mathbb{D}$;

(ii) $\quad \operatorname{ber}\left(f^{2}(A)\right)+\operatorname{ber}\left(f^{4}(A)\right)^{2}+\operatorname{ber}\left(f^{3}(A)\right)^{2}\left(\operatorname{ber}\left(\left(I-f^{2}(A)\right)^{\frac{1}{2}}\right)\right)^{2}$

$$
\geq \sup _{\lambda \in \mathbb{D}}\left[\widetilde{f^{2}(A)}(\lambda) \widetilde{f^{4}(A)}(\lambda)\right] \text {. }
$$

(iii) If $\left\|I-f^{2}(A)\right\|<1$, then

$$
\operatorname{ber}\left(f^{4}(A)\right)^{2}+\operatorname{ber}\left(f^{3}(A)\right)^{2} \geq \operatorname{ber}\left(f^{2}(A)\right)\left(\widetilde{c}\left(f^{4}(A)-1\right) .\right.
$$

Proof. (i) We set

$$
\mathcal{D}:=\left\{(\lambda, \mu) \in \mathbb{D} \times \mathbb{D}: \operatorname{Re}(\lambda \bar{\mu})=\frac{1}{2}\right\} .
$$

Putting now $H=H^{2}(\mathbb{D})$ (the Hardy space), $x=\widehat{k}_{\lambda}, y=\widehat{k}_{\mu}$ and $e=\mathbb{I}$ in the formula (5.1) with $(\lambda, \mu) \in \mathcal{D}$, we have 


$$
\begin{aligned}
& 1-\left|\left\langle\widehat{k}_{\lambda}, \widehat{k}_{\mu}\right\rangle\right|^{2} \geq\left(\operatorname{det}\left[\begin{array}{cc}
\left\langle\widehat{k}_{\lambda}, \mathbb{I}\right\rangle & \left(1-\left|\left\langle\widehat{k}_{\lambda}, \mathbb{I}\right\rangle\right|^{2}\right)^{\frac{1}{2}} \\
\left\langle\widehat{k}_{\mu}, \mathbb{I}\right\rangle & \left(1-\left|\left\langle\widehat{k}_{\mu}, \mathbb{I}\right\rangle\right|^{2}\right)^{\frac{1}{2}}
\end{array}\right]\right)^{2} \\
& =\left(\operatorname{det}\left[\begin{array}{ll}
\left(1-|\lambda|^{2}\right) & \left(1-\left(1-|\lambda|^{2}\right)\right)^{\frac{1}{2}} \\
\left(1-|\mu|^{2}\right) & \left(1-\left(1-|\mu|^{2}\right)\right)^{\frac{1}{2}}
\end{array}\right]\right)^{2} \\
& =\left(\operatorname{det}\left[\begin{array}{ll}
\left(1-|\lambda|^{2}\right)^{\frac{1}{2}} & |\lambda| \\
\left(1-|\mu|^{2}\right)^{\frac{1}{2}} & |\mu|
\end{array}\right]\right)^{2} \\
& =\left(|\mu|\left(1-|\lambda|^{2}\right)^{\frac{1}{2}}-|\lambda|\left(1-|\mu|^{2}\right)^{\frac{1}{2}}\right)^{2} \\
& =|\lambda|^{2}+|\mu|^{2}-2|\lambda|^{2}|\mu|^{2}-2|\lambda||\mu|\left(1-|\lambda|^{2}\right)^{\frac{1}{2}}\left(1-|\mu|^{2}\right)^{\frac{1}{2}},
\end{aligned}
$$

hence

$$
\begin{aligned}
& 1-\left|\left\langle\frac{\left(1-|\lambda|^{2}\right)^{\frac{1}{2}}}{1-\bar{\lambda} z}, \frac{\left(1-|\mu|^{2}\right)^{\frac{1}{2}}}{1-\bar{\mu} z}\right\rangle\right|^{2} \\
& \quad \geq|\lambda|^{2}+|\mu|^{2}-2|\lambda|^{2}|\mu|^{2}-2|\lambda||\mu|\left(1-|\lambda|^{2}\right)^{\frac{1}{2}}\left(1-|\mu|^{2}\right)^{\frac{1}{2}}
\end{aligned}
$$

that is

$$
\begin{aligned}
& 1-\frac{\left(1-|\lambda|^{2}\right)\left(1-|\mu|^{2}\right)}{|1-\bar{\lambda} \mu|^{2}} \\
& \quad \geq|\lambda|^{2}+|\mu|^{2}-2|\lambda|^{2}|\mu|^{2}-2|\lambda||\mu|\left(1-|\lambda|^{2}\right)^{\frac{1}{2}}\left(1-|\mu|^{2}\right)^{\frac{1}{2}},
\end{aligned}
$$

and thus

$$
\begin{aligned}
& \frac{1-2 \operatorname{Re}(\lambda \bar{\mu})+|\lambda|^{2}+|\mu|^{2}}{1-2 \operatorname{Re}(\lambda \bar{\mu})+|\lambda|^{2}|\mu|^{2}} \\
& \quad \geq|\lambda|^{2}+|\mu|^{2}-2|\lambda|^{2}|\mu|^{2}-2|\lambda||\mu|\left(1-|\lambda|^{2}\right)^{\frac{1}{2}}\left(1-|\mu|^{2}\right)^{\frac{1}{2}} .
\end{aligned}
$$


Since $(\lambda, \mu) \in \mathcal{D}$, we have that $1-2 \operatorname{Re}(\lambda \bar{\mu})=0$, and hence

$$
\frac{|\lambda|^{2}+|\mu|^{2}}{|\lambda|^{2}|\mu|^{2}} \geq|\lambda|^{2}+|\mu|^{2}-2|\lambda|^{2}|\mu|^{2}-2|\lambda||\mu|\left(1-|\lambda|^{2}\right)^{\frac{1}{2}}\left(1-|\mu|^{2}\right)^{\frac{1}{2}}
$$

whence

$$
\begin{aligned}
|\lambda|^{2}+ & |\mu|^{2}+2|\lambda|^{4}|\mu|^{4}+2|\lambda|^{3}|\mu|^{3}\left(1-|\lambda|^{2}\right)\left(1-|\mu|^{2}\right)^{\frac{1}{2}} \\
& \geq|\lambda|^{4}|\mu|^{2}+|\lambda|^{2}|\mu|^{4}
\end{aligned}
$$

for any pair $(\lambda, \mu) \in \mathcal{D}$. We let $|\lambda|=a>0,|\mu|=b>0$. Since $(\lambda, \mu) \in \mathcal{D}$ is arbitrary, the numbers $a$ and $b$ are also arbitrary. Therefore there exist $x, y \in J$ such that $f(x)=a$ and $g(y)=b$. Then, we have from the last inequality that

$$
\begin{aligned}
f^{2}(x) & +g^{2}(y)+2 f^{4}(x) g^{4}(y)+2 f^{3}(x) g^{3}(y)\left(\left(1-f^{2}(x)\right)\left(1-g^{2}(y)\right)\right)^{\frac{1}{2}} \\
& \geq f^{4}(x) g^{2}(y)+f^{2}(x) g^{4}(y)
\end{aligned}
$$

for all $x, y \in J$. From this inequality, by using Kian's method [27], we have

$$
\begin{aligned}
f^{2}(A) & +g^{2}(y)+2 f^{4}(A) g^{4}(y)+2 f^{3}(A) g^{3}(y)\left(\left(1-f^{2}(A)\right)\left(1-g^{2}(y)\right)\right)^{\frac{1}{2}} \\
& \geq f^{4}(A) g^{2}(y)+f^{2}(A) g^{4}(y)
\end{aligned}
$$

for all $y \in J$ and for any self adjoint operator $A \in \mathcal{B}\left(H^{2}\right)$ with $\sigma(A) \subset J$ such that $I-f^{2}(A) \geq 0$. It follows from 5.2 that

$$
\begin{aligned}
\left\langle f^{2}(A) \widehat{k}_{\lambda}, \widehat{k}_{\lambda}\right\rangle & +g^{2}(y)+2 g^{4}(y)\left\langle f^{4}(A) \widehat{k}_{\lambda}, \widehat{k}_{\lambda}\right\rangle \\
& +2 g^{3}(y)\left\langle f^{3}(A) \widehat{k}_{\lambda}, \widehat{k}_{\lambda}\right\rangle\left(\left(1-g^{2}(y)\right)^{\frac{1}{2}}\left\langle\left(I-f^{2}(A)\right)^{\frac{1}{2}} \widehat{k}_{\lambda}, \widehat{k}_{\lambda}\right\rangle\right) \\
\geq & g^{2}(y)\left\langle f^{4}(A) \widehat{k}_{\lambda}, \widehat{k}_{\lambda}\right\rangle+g^{4}(y)\left\langle f^{2}(A) \widehat{k}_{\lambda}, \widehat{k}_{\lambda}\right\rangle
\end{aligned}
$$

or equivalently,

$$
\begin{aligned}
\widetilde{f^{2}(A)}(\lambda) & +g^{2}(y)+2 g^{4}(y) \widetilde{f^{4}(A)}(\lambda) \\
& +2 g^{3}(y) \widetilde{f^{3}(A)}(\lambda)\left(1-g^{2}(y)\right)^{\frac{1}{2}} \widehat{\left(I-f^{2}(A)\right)^{\frac{1}{2}}}(\lambda) \\
\geq & g^{2}(y) \widetilde{f^{4}(A)}(\lambda)+g^{4}(y) \widetilde{f^{2}(A)}(\lambda), \quad \text { for each } \lambda \in \mathbb{D} .
\end{aligned}
$$


Now, by applying the functional calculus for a self-adjoint operator $B \in \mathcal{B}\left(H^{2}\right)$ with spectrum in $J$ with respect to the variable $y$, we get

$$
\begin{aligned}
\widetilde{f^{2}(A)}(\lambda) & +\left\langle g^{2}(B) \widehat{k}_{\mu}, \widehat{k}_{\mu}\right\rangle+2 \widetilde{f^{4}(A)}(\lambda)\left\langle g^{4}(B) \widehat{k}_{\mu}, \widehat{k}_{\mu}\right\rangle \\
& +2 \widetilde{f^{3}(A)(\lambda)}\left\langle g^{3}(B) \widehat{k}_{\mu}, \widehat{k}_{\mu}\right\rangle \widetilde{\left(I-f^{2}(A)\right)^{\frac{1}{2}}}(\lambda) \widetilde{\left(I-g^{2}(B)\right)^{\frac{1}{2}}}(\mu) \\
\geq & \widetilde{g^{2}(B)}(\mu) \widetilde{f^{4}(A)}(\lambda)+\widetilde{g^{4}(B)}(\mu) \widetilde{f^{2}(A)}(\lambda)
\end{aligned}
$$

that is

$$
\begin{aligned}
\widetilde{f^{2}(A)}(\lambda) & +\widetilde{g^{2}(B)}(\mu)+2 \widetilde{f^{4}(A)}(\lambda) \widetilde{g^{4}(B)}(\mu) \\
& +\widetilde{2 f^{3}(A)}(\lambda) \widetilde{g^{3}(B)}(\mu) \widetilde{\left(I-f^{2}(A)\right)^{\frac{1}{2}}}(\lambda) \widetilde{\left(I-g^{2}(B)\right)^{\frac{1}{2}}}(\mu) \\
\geq & \widetilde{g^{2}(B)}(\mu) \widetilde{f^{4}(A)}(\lambda)+\widetilde{g^{4}(B)}(\mu) \widetilde{f^{2}(A)}(\lambda)
\end{aligned}
$$

for all $\lambda, \mu \in \mathbb{D}$ and every pair of self-adjoint operators $A, B \in \mathcal{B}\left(H^{2}\right)$ with spectra in $J$ such that $I-f^{2}(A)$ and $I-g^{2}(B)$ are positive. This proves (i).

(ii) We particularly obtain from inequality (5.3) for $B=A, g=f$ and $\mu=\lambda$ that

$$
\begin{aligned}
& \widetilde{2 f^{2}(A)}(\lambda)+2\left[\widetilde{f^{4}(A)}(\lambda)\right]^{2}+2\left[\widetilde{f^{3}(A)}(\lambda)\right]^{2}\left[\widetilde{\left(I-f^{2}(A)\right)^{\frac{1}{2}}}(\lambda)\right]^{2} \\
& \geq 2 \widetilde{f^{2}(A)}(\lambda) \widetilde{f^{4}(A)}(\lambda)
\end{aligned}
$$

and thereby, we obtain that

$$
\begin{aligned}
& \operatorname{ber}\left(f^{2}(A)\right)+\left(\operatorname{ber}\left(f^{4}(A)\right)\right)^{2}+\left(\operatorname{ber}\left(f^{3}(A)\right)\right)^{2}\left(\operatorname{ber}\left(I-f^{2}(A)\right)^{\frac{1}{2}}\right)^{2} \\
& \quad \geq \sup _{\lambda \in \mathcal{D}}\left[\widetilde{f^{2}(A)}(\lambda) \widetilde{f^{4}(A)}(\lambda)\right]
\end{aligned}
$$

which proves (ii).

(iii) Is an immediate consequence of inequality (5.4). Indeed, the operator $f^{2}(A)$ is invertible because $\left\|I-f^{2}(A)\right\|<1$. Then

$$
\left\|f^{2}(A) \widehat{k}_{\lambda}\right\| \geq \frac{1}{\left\|\left(f^{2}(A)\right)^{-1}\right\|}\left\|\widehat{k}_{\lambda}\right\|=\left\|\left(f^{2}(A)\right)^{-1}\right\|^{-1}
$$


and ber $\left(I-f^{2}(A)\right)^{1 / 2} \leq\left\|I-f^{2}(A)\right\|<1$, hence

$$
\begin{aligned}
\left\langle f^{2}(A) \widehat{k}_{\lambda}, f^{2}(A) \widehat{k}_{\lambda}\right\rangle^{1 / 2} & =\left\langle\left(f^{2}(A)\right)^{*} f^{2}(A) \widehat{k}_{\lambda}, \widehat{k}_{\lambda}\right\rangle^{1 / 2} \\
& =\left\langle f^{4}(A) \widehat{k}_{\lambda}, \widehat{k}_{\lambda}\right\rangle \text { (since } f^{2}(A) \text { is self-adjoint) } \\
& =\widetilde{f^{4}(A)}(\lambda) \geq\left\|\left(f^{2}(A)\right)^{-1}\right\|^{-1},
\end{aligned}
$$

which means that $\widetilde{c}\left(f^{4}(A)\right) \geq\left\|\left(f^{2}(A)\right)^{-1}\right\|^{-1}>0$. Therefore, we obtain from (5.4) the desired inequality, which proves (iii).

Note that the Berezin symbol is not multiplicative, i.e., $\widetilde{A B}(\lambda) \neq \widetilde{A}(\lambda) \widetilde{B}(\lambda)$ in general, see Kiliç [28].

\section{ACKNOWLEDGEMENTS}

The authors thank to the referee for his/her useful remarks. Also, the first and second authors would like to extend his sincere appreciation to the Deanship of Scientific Research at King Saud University for its funding of this research through the Research Group Project no. RGPVPP-323. This paper was supported by Süleyman Demirel University project no FYL-2018-6962.

\section{REFERENCES}

[1] T. Ando, C.-K. Li, R. Mathias, Geometric means, Linear Algebra Appl. 385 (2004), 305-334.

[2] L. Arambas̆IĆ, D. Bakić, M.S. Moslehian, A treatment of the Cauchy-Schwarz inequality in $C^{*}$-Modules, J. Math. Anal. Appl. 381 (2011), $546-556$.

[3] N. AronZajn, Theory of reproducing kernels, Trans. Amer. Math. Soc. 68 (1950), 337-404.

[4] M. BAKHeRAD, Some Berezin number inequalities for operator matrices, Czechoslovak Math. J., 68 (4) (2018), 997-1009.

[5] M. BAKherad, M. GARAYev, Berezin number inequalities for operators, Concr. Oper. 6 (1) (2019), 33-43.

[6] H. Başaran, M. Gürdal, A.N. GünCAN, Some operator inequalities associated with Kantorovich and Hölder-McCarthy inequalities and their applications, Turkish J. Math. 43 (1) (2019), 523-532.

[7] F.A. Berezin, Covariant and contravariant symbols for operators, Math. USSR-Izv. 6 (1972), 1117-1151. 
[8] F.A. BerezIn, Quantization, Math. USSR-Izv. 8 (1974), 1109-1163.

[9] L.A. Coburn, Berezin transform and Weyl-type unitary operators on the Bergman space, Proc. Amer. Math. Soc. 140 (2012), 3445-3451.

[10] S.S. Dragomir, New inequalities of the Kantorovich type for bounded linear operators in Hilbert spaces, Linear Algebra Appl. 428 (11-12) (2008), $2750-2760$.

[11] S.S. DraGomiR, Improving Schwartz inequality in inner product spaces, Linear Multilinear Algebra 67 (2) (2019), 337-347.

[12] M. Enомото, Commutative relations and related topics, Kyoto University, 1039 (1998), $135-140$.

[13] T. FurutA, Operator inequalities associated with Hölder-McCarthy and Kantorovich inequalities, J. Inequal. Appl. 2 (2) (1998), 137-148.

[14] T. FurutA, "Invitation to Linear Operators. From matrices to bounded linear operators on a Hilbert space", Taylor \& Francis, London, 2001 (p. 266).

[15] T. Furuta, J. Mićić Hot, J.E. PeČarić, Y. Seo, Mond-Pecaric method in operator inequalities, in "Inequalities for bounded selfadjoint operators on a Hilbert space", Monographs in Inequalities, Vol. 1, Element, Zagreb, 2005.

[16] M.T. Garayev, M. Gürdal, M.B. Huban, Reproducing kernels, Engliš algebras and some applications, Studia Math. 232 (2) (2016), 113-141.

[17] M.T. Garayev, M. Gürdal, A. Okudan, Hardy-Hilbert's inequality and power inequalities for Berezin numbers of operators, Math. Inequal. Appl. 19 (3) (2016), 883-891.

[18] M.T. Garayev, M. Gürdal, S. Saltan, Hardy type inequality for reproducing kernel Hilbert space operators and related problems, Positivity 21 (4) (2017), 1615-1623.

[19] M.T. Garayev, Berezin symbols, Hölder-McCarthy and Young inequalities and their applications, Proc. Inst. Math. Mech. Natl. Acad. Sci. Azerb. 43 (2) (2017), 287-,295.

[20] W. Greub, W. Rheinboldt, On a generalization of an inequality of L.V. Kantorovich, Proc. Amer. Math. Soc. 10 (1959), 407-415.

[21] M. Gürdal, M.T. Garayev, S. Saltan, U. Yamanci, On some numerical characteristics of operators, Arab J. Math. Sci. 21 (1) (2015), 118-126.

[22] P. Halmos, "A Hilbert Space Problem Book", Graduate Texts in Mathematics, Vol. 19, Springer-Verlag, New York-Berlin, 1982.

[23] K. Hoffman, "Banach Spaces of Analytic Functions", Dover Publications, Mineola, New York, 2007.

[24] L.V. Kantorović, Functional analysis and applied mathematics, Uspehi Matem. Nauk (N.S.) 3(6(28)) (1948), 89-185 (in Russian).

[25] M.T. KARAEV, Reproducing kernels and Berezin symbols techniques in various questions of operator theory, Complex Anal. Oper. Theory 7 (4) (2013), $983-1018$.

[26] M.T. Karaev, S. Saltan, Some results on Berezin symbols, Complex Var. Theory Appl. 50 (2005), 185-193. 
[27] M. KIAN, Hardy-Hilbert type inequalities for Hilbert space operators, Ann. Funct. Anal. 3 (2) (2012), 129-135.

[28] S. KILIÇ, The Berezin symbol and multipliers of functional Hilbert spaces, Proc. Amer. Math. Soc. 123 (1995), 3687-3691.

[29] E.C. Lance, "Hilbert $C^{*}$-modules", London Math. Soc. Lecture Notes Series, 210, Cambridge University Press, Cambridge, 1995.

[30] S. Liu, H. NeudeCKeR, Several matrix Kantorovich-type inequalities, J. Math. Anal. Appl. 197 (1996), 23-26.

[31] V.M. Manullov, E.V. Troitsky, "Hilbert $C^{*}$-modules", Translations of Mathematical Monographs, 226, American Mathematical Society, Providence RI, , 2005.

[32] A.W. Marshall, I. Olkin, Matrix versions of the Cauchy and Kantorovich inequalities, Aequationes Math. 40 (1) (1990), 89-93.

[33] D.S. Mitrinovic, J.E. PeČarić, A.M. Fink, "Classical and new inequalities in analysis", Kluwer Academic Publishers Group, Dordrecht, 1993.

[34] M.S. Moslehian, Recent developments of the operator Kantorovich inequality, Expo. Math. 30 (2012), 376-388.

[35] R. NAKAмото, M. NAKAmURA, Operator mean and Kantorovich inequality, Math. Japon. 44 (3) (1996), 495-498.

[36] M. NiezGodA, Kantorovich type inequalities for ordered linear spaces, Electron. J. Linear Algebra 20 (2010), 103-114.

[37] C. Pearcy, An elementary proof of the power inequality for the numerical radius, Michigan Math. J. 13 (1966), 289-291.

[38] G. Strang, On the Kantorovich inequality, Proc. Amer. Math. Soc. 11 (1960), 468.

[39] U. Yamanci, M.T. Garayev, C. Çelik, Hardy-Hilbert type inequality in reproducing kernel Hilbert space: its applications and related results, Linear Multilinear Algebra 67 (4) (2019), 830-842.

[40] U. Yamanci, M. Gürdal, M.T. GaraYev, Berezin number inequality for convex function in reproducing kernel Hilbert space, Filomat 31 (18) (2017), $5711-5717$.

[41] T. YAMAZAKI, An extension of Kantorovich inequality to $n$-operators via the geometric mean by Ando-Li-Mathias, Linear Algebra Appl. 416 (2-3) (2006), $688-, 695$.

[42] T. Yamazaki, M. YANAGIDA, Characterizations of chaotic order associated with Kantorovich inequality, Sci. Math. 2 (1) (1999), 37-50.

[43] H. Umegaki, Conditional expectation in an operator algebra, Tohoku Math. J. (2) 6 (1954), $177-181$.

[44] F. Zhang, Equivalence of the Wielandt inequality and the Kantorovich inequality, Linear Multilinear Algebra 48 (3) (2001), 275-279. 